\title{
Positive Switched System Approach to Traffic Signal Control for Oversaturated Intersection
}

\author{
Lin $\mathrm{Du}^{1}$ and Yun Zhang ${ }^{2}$ \\ ${ }^{1}$ School of Transportation and Logistics, Southwest Jiaotong University, Chengdu 610031, China \\ ${ }^{2}$ Mianyang Vocational and Technical College, Mianyang 621000, China \\ Correspondence should be addressed to Lin Du; 6473318@qq.com
}

Received 5 June 2014; Accepted 22 July 2014; Published 17 August 2014

Academic Editor: Weiming Xiang

Copyright (C) 2014 L. Du and Y. Zhang. This is an open access article distributed under the Creative Commons Attribution License, which permits unrestricted use, distribution, and reproduction in any medium, provided the original work is properly cited.

The online traffic signalization for intersection is addressed. A new model for intersection called positive switched system is presented. Then, based on the dissipativity analysis results for positive switched system, an online state-feedback control strategy for traffic signal in two-phase intersection section is proposed. A numerical example is provided to illustrate the effectiveness of our theoretical findings. Finally, in order to extend to more general cases, multiphase intersection is considered, and general dissipativity-based control is presented.

\section{Introduction}

Traffic signal control is a long-lasting research problem in urban transportation network system [1-3]. The effectiveness of a traffic signal system can reduce the incidence of delays, stops, fuel consumption, emission of pollutants, and accidents. Moreover, due to the rapid growth of traffic congestion, an effective traffic signalization plays an important role of relieving the oversaturated situation such as related articles $[1,4-11]$ and references cited therein. Most of the signal control strategies are based on fixed-time signal control $[1,4-7]$. However, since the fixed-time strategies are based on historical data rather than real-time data, they are only applicable in undersaturated traffic conditions. In a few recent papers, some online signalization methods have been proposed [2, 8-13], which are more adaptive to the realtime traffic conditions. As for oversaturated condition, some significant results have been reported. For example, in [9], an optimal traffic light switching scheme was presented. Generally, it resorts to a minimization problem over a set of an extended linear complementarity problem, which is not an easy task when switching cycles is large. And concerned with recent notable result [10], a dissipative idea is applied into traffic signal design problem; a state feedback controller based on dissipativity-based control is derived. This dissipative approach provides a new insight on traffic signalization problem and intersection system. In our paper, we follow the dissipative idea and further exploit the positivity and switched properties of intersection system, which leads to a positive switched system approach into the dissipativitybased control framework. The particular class of hybrid system called positive switched system is used to model the intersection system.

Switched system can be efficiently used to model many practical systems which are inherently multimodel in the sense that several dynamical systems are required to describe their behavior. For more details of the recent results on the basic problems in stability and stabilization for switched systems, the reader is referred to survey papers [14-24] and the references cited therein. Moreover, positive switched system refers to the variables of system which are always confined to the positive orthant. Due to the obvious switched and positive characteristic of intersection system with traffic signal, the positive switched system is an appropriate model for it, but as far as we know, there exists no result based on the model of positive switched system. Thus, in our paper, we first set up a positive switched system model for two-phase intersection system, which is shown to be able to be modeled as a positive switched system with two subsystems. Then, a dissipativity-based control strategy is proposed by solving a set of linear programming (LP) problem which can be efficiently solved with aid of existing software toolbox. Then, 


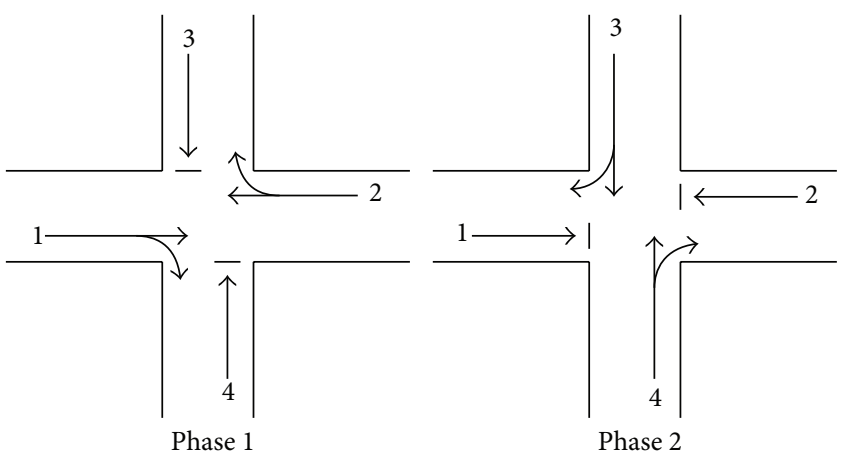

FIGURE 1: Intersection with four approaches and two-phase signal.

an extension from two-phase intersection to multiphase intersection system is presented to meet more general cases.

The rest of this paper is organized as follows. In Section 2 the system model and problem formulation are introduced; dissipativity analysis for positive switched system is proposed in Section 3. The dissipativity-based control solution for twophase intersection is given in Section 4. In Section 5 the results are extended to multiphase cases. Conclusions are given in Section 6.

Notations. The notations used in this paper are fairly standard. The superscript " $T$ " stands for matrix transposition, $\mathbb{R}^{n}$ denotes the $n$ dimensional Euclidean space, and $\mathbb{N}$ represents the set of nonnegative integers. In addition, in symmetric block matrices, we use $*$ as an ellipsis for the terms that are introduced by symmetry. The notation $A>0(A \succeq 0)$ means all the elements $a_{i j}>0\left(a_{i j} \geq 0\right)$, where $a_{i j}$ denotes the element in the $(i, j)$ position of $A$.

\section{System Description and Problem Formulation}

2.1. System Description and Modeling. The urban transportation system is composed of a network of intersections. Generally, an intersection is operated by a traffic signal that decides the movements of vehicles to pass the intersections or to stop to generate the queues. The movement may include vehicles going straight, turning left, turning right, or a combination of them.

In order to show our control idea clearly, we first consider the single intersection with four approaches and the traffic signal which has two phases, which are illustrated by Figure 1.

It is noted that the movements 1 and 2 are supposed to have same characteristics, and same consideration holds for movements 3 and 4 .

Considering the transportation status at the time of the traffic signal turning from one phase to another, and denoting the time instant as $k, k \in \mathbb{N}$, we are going to model the intersection system described in Figure 1; several useful definitions are introduced as follows.

Green Time $g_{p}(k)$. It is the time for the movements for the successively activated phase $p, p \in \mathscr{P}=\{1,2\}$, which is the control input signal required to be determined at each instant $k$ for the intersection. It is assumed that there exists a $g_{p \text {,max }}$ such that $0 \leq g_{p}(k) \leq g_{p \text {, max }}$.

Lost Time L. Lost time is defined as a period that is not used effectively in each phase by incoming traffic flow through an intersection, such as the start-up delay. Lost time is generally considered constant.

Effective Green Time $g_{p}^{e}(k)$. It is the time actually available for movement for phase $p$. Obviously, we can obtain $g_{p}^{e}(k)+L=$ $g_{p}(k), p \in \mathscr{P}=\{1,2\}$.

Input Flow Rate $q_{i}$. It is the input flow rate for movement $i$, $i \in \mathscr{I}=\{1,2,3,4\}$.

Saturation Flow Rate $s_{i}$. It is the saturation flow rate for the movement $i, i \in \mathscr{I}=\{1,2,3,4\}$, which is defined as the maximum number of vehicles being able to use the intersection without interruption during the effective green time.

The Number of Arrivals $A_{i, T}$. It is the number of vehicles joining the movement $i$ during the time $T$. It can be figured out as $A_{i, T}=q_{i} T$.

The Number of Departures $D_{i, T}$. It is the number of vehicles departing from the movement $i$ during the time $T$, which can be calculated by $D_{i, T}=s_{i} T$.

Queue Length $x_{i}(k)$. It is the queue length of movement $i$, $i \in \mathscr{I}$, at time instant $k$. It is assumed that the value of queue length $x_{i}$ (number of vehicles) can be measured in real time, which can be obtained when video detection systems are utilized; otherwise, the local occupancy measurements $o_{i}$, collected in real time by traditional detector loops, can be transformed into (approximate) numbers of vehicles via suitable nonlinear functions $x_{i}=f_{i}\left(o_{i}\right)$ such as in [3].

Moreover, since the congestion situation is not considered in our model, the queue lengths are always beneath their capacities, which is denoted by $x_{i, \max }, i \in \mathscr{I}$. Thus, queue length $x_{i}$ belongs to the region of admissible states as $X_{i}=$ $\left\{x \mid 0 \leq x_{i} \leq x_{i, \max }\right\}$.

Based on the above definitions, we are now in the position to model the intersection system. Since there are two phases, it is natural that there are two dynamics for Phases 1 and 2, respectively.

Phase 1. The queue length $x_{i}(k)$ evolves according to $x_{i}(k+$ $1)=x_{i}(k)+A_{i, g_{1}}-D_{i, g_{1}}$. For movements 1 and 2 which have same characteristics, it is obtained that $A_{i, g_{1}}=q_{i} g_{1}, i=1,2$, and $D_{i, g_{1}}=s_{i} g_{1}^{e}=s_{i}\left(g_{1}-L\right), i=1,2$; we have the following equation in Phase 1:

$$
x_{i}(k+1)=x_{i}(k)+\left(q_{i}-s_{i}\right) g_{1}+s_{i} L, \quad i=1,2 .
$$


And for movements 3 and 4 , since the movement is stopped which implies $A_{i, g_{1}}=q_{i} g_{1}, i=3,4$, and $D_{i, g_{1}}=0$, $i=3,4$, it is obtained that

$$
x_{i}(k+1)=x_{i}(k)+q_{i} g_{1}, \quad i=3,4 .
$$

Phase 2. Following the same guideline in Phase 1, the following evolution equations can be derived:

$$
\begin{gathered}
x_{i}(k+1)=x_{i}(k)+q_{i} g_{2}, \quad i=1,2, \\
x_{i}(k+1)=x_{i}(k)+\left(q_{i}-s_{i}\right) g_{2}+s_{i} L, \quad i=3,4 .
\end{gathered}
$$

Among the two subsystems concerned with two phases, there is a switching signal $\sigma(k)$ determining which subsystem is activated at each instant $k$. Define

$$
\eta_{p}(k)= \begin{cases}1 & \sigma(t)=p \\ 0 & \text { otherwise }\end{cases}
$$

Augmenting the dynamics in Phases 1 and 2, the above equations can be restated as a switched system composed of two subsystems in state space form

$$
\begin{array}{r}
\mathbf{x}(k+1)=\sum_{p=1}^{2} \eta_{p}(k)\left[\mathbf{x}(k)+\mathbf{B}_{p} u_{p}(k)+\mathbf{C}_{p}\right], \\
0 \leq u_{p}(k) \leq g_{p, \max },
\end{array}
$$

where

$$
\begin{gathered}
\mathbf{x}(k)=\left[\begin{array}{c}
x_{1}(k) \\
x_{2}(k) \\
x_{3}(k) \\
x_{4}(k)
\end{array}\right], \quad \mathbf{B}_{1}=\left[\begin{array}{c}
q_{1}-s_{1} \\
q_{2}-s_{2} \\
q_{3} \\
q_{4}
\end{array}\right], \\
\mathbf{B}_{2}=\left[\begin{array}{c}
q_{1} \\
q_{2} \\
q_{3}-s_{3} \\
q_{4}-s_{4}
\end{array}\right], \quad \mathbf{C}_{1}=\left[\begin{array}{c}
s_{1} L \\
s_{2} L \\
0 \\
0
\end{array}\right], \quad \mathbf{C}_{2}=\left[\begin{array}{c}
0 \\
0 \\
s_{3} L \\
s_{4} L
\end{array}\right], \\
u_{1}(k)=g_{1}(k), \quad u_{2}(k)=g_{2}(k) .
\end{gathered}
$$

Obviously, since the phases work in turns in the intersection system model, the switching signal $\sigma(k)$ is defined as

$$
\sigma(k)=p \quad \text { if } \sigma(k-1)=q, \quad \text { where } p \neq q, p, q=1,2,
$$

which implies that the switching occurs at each instant $k$.

The most reported model is considered to have a fixed cycle (one repetition of the basic series of signal phases combinations at a junction), which has to be prespecified appropriately, and an inappropriate choice of cycle could lead to a bad control performance. On the other hand, there is no cycle time constraint in our switched system model, or no cycle time has to be designed previously; only controllable green time needs to be considered.
2.2. Control Problem Formulation. The purpose of our control is to relieve the oversaturated queue by the traffic signal. With the controllable green time $g_{p}, p \in \mathscr{P}=\{1,2\}$, we are going to design a state feedback controller in the form of

$$
u_{p}(k)=\sum_{p=1}^{2} \eta_{p}(k) \mathbf{K}_{p} \mathbf{x}(k), \quad 0 \leq u_{p}(k) \leq g_{p, \max },
$$

where $\mathbf{K}_{p}, p \in \mathscr{P}$, is the feedback gains needed to be determined. Substituting state feedback controller (8) into system model (5), the closed loop of intersection system is expressed as

$$
\begin{array}{r}
\mathbf{x}(k+1)=\sum_{p=1}^{2} \eta_{p}(k)\left[\mathbf{A}_{p} \mathbf{x}(k)+\mathbf{C}_{p}\right], \\
0 \leq \mathbf{K}_{p} \mathbf{x}(k) \leq g_{p, \text { max }}
\end{array}
$$

where $\mathbf{A}_{p}=\mathbf{I}+\mathbf{B}_{p} \mathbf{K}_{p}$. Under the control of (8), our design objective is to render the system (9) nonaccumulative with flux supply rate.

At first, the lengths of queue $x_{i}(k), \forall k \in \mathbb{N}$, are supposed to be nonnegative in the evolution according to the system dynamics given any initial $x_{i}(0) \geq 0$. Thus, to ensure the availability of designed state feedback controller, the positivity of $\mathbf{x}(k)$ has to be preserved during the time; that is,

$$
\mathbf{x}(k) \geq 0, \quad \forall k \in \mathbb{N} .
$$

Then, to make the system nonaccumulative, a particular copositive storage function indicating the total lengths of the movement stored in the system is introduced as

$$
S(k)=\mathbf{e}^{T} \mathbf{x}(k)
$$

where $\mathbf{e}^{T}=\left[\begin{array}{llll}1 & 1 & 1 & 1\end{array}\right]$. The flow of vehicles supplied to the system is the total arrivals in each phase described by

$$
A_{p}(k)=g_{p}(k) \sum_{i=1}^{4} q_{i}=\mathbf{K}_{p} \mathbf{x}(k) \sum_{i=1}^{4} q_{i}, \quad p \in \mathscr{P} .
$$

And the total of departures in Phase 1 is $D_{1}=\left(s_{1}+s_{2}\right)\left(g_{1}-\right.$ $L)=\left(s_{1}+s_{2}\right) g_{1}-\left(s_{1}+s_{2}\right) L$ and $D_{2}=\left(s_{3}+s_{4}\right)\left(g_{2}-L\right)=$ $\left(s_{3}+s_{4}\right) g_{2}-\left(s_{3}+s_{4}\right) L$ is for Phase 2 . Thus, the flow of vehicles extracted from the system is

$$
D_{p}(k)=d_{p} g_{p}-d_{p} L=d_{p} \mathbf{K}_{p} \mathbf{x}(k)-d_{p} L, \quad p \in \mathscr{P},
$$

where $d_{1}=s_{1}+s_{2}$ and $d_{2}=s_{3}+s_{4}$. The flux supply rate in each phase can be defined as

$$
\theta(k)=\sum_{p=1}^{2} \eta_{p}(k) \theta_{p}(k)=\sum_{p=1}^{2} \eta_{p}(k)\left[A_{p}(k)-D_{p}(k)\right]
$$

that is,

$$
\theta(k)=\sum_{p=1}^{2} \eta_{p}(k) \theta_{p}(k)=\sum_{p=1}^{2} \eta_{p}(k)\left[\boldsymbol{\alpha}_{p} \mathbf{x}(k)+\beta_{p}\right],
$$


where $\boldsymbol{\alpha}_{p}=\mathbf{K}_{p}\left(\sum_{i=1}^{4} q_{i}-d_{p}\right)$ and $\beta_{p}=d_{p} L$. To achieve a nonaccumulative system with storage function $S(k)$ and supply rate $\theta(k)$, the following condition has to be satisfied:

$$
\Delta S(k)=S(k+1)-S(k) \leq \theta(k),
$$

which implies that the variation in the total queue length of the system is always less than or equal to the input flux minus the output flux. In this case the system with feedback controller (8) is nonaccumulative with respect to the flux supply rate $\theta(k)$.

\section{Dissipativity Analysis for Positive Switched System}

In this section, we will investigate the dissipativity of switched positive system, which plays the key role in solving the control problem for intersection system.

Consider the following switched positive system composed by $P$ subsystems:

$$
\mathbf{x}(k+1)=\sum_{p=1}^{P} \eta_{p}(k)\left[\mathbf{A}_{p} \mathbf{x}(k)+\mathbf{C}_{p}\right]
$$

where $\mathbf{x}(k) \in \mathbb{R}^{n}$ is the state vector, $\mathbf{A}_{p}$ and $\mathbf{C}_{p}$ are constant matrices with appropriate dimensions, and the switching law among subsystems is considered arbitrary.

Definition 1. System (1) is said to be positive if and only if, for any initial condition $\mathbf{x}(0) \succeq 0$, the corresponding trajectory $\mathbf{x}(k) \geq 0$ holds for all $k \in \mathbb{N}$.

Lemma 2. System (1) is positive if and only if $\mathbf{A}_{p} \geq 0$ and $\mathbf{C}_{p} \geq$ $0, \forall p \in \mathscr{P}$.

Proof. The sufficient part is obvious. We consider the necessity. Denote

$$
\begin{aligned}
& \mathbf{x}(k)=\left[\begin{array}{llll}
x_{1}(k) & x_{2}(k) & \cdots & x_{n}(k)
\end{array}\right]^{T}, \\
& \mathbf{A}_{p}=\left[\begin{array}{cccc}
a_{1,1} & a_{1,2} & \cdots & a_{1, n} \\
a_{2,1} & a_{1,2} & \cdots & a_{2 n} \\
\vdots & \vdots & \ddots & \vdots \\
a_{n, 1} & a_{n, 2} & \cdots & a_{n, n}
\end{array}\right], \quad \mathbf{C}_{p}=\left[\begin{array}{c}
c_{1} \\
c_{2} \\
\vdots \\
c_{n}
\end{array}\right]
\end{aligned}
$$

we have

$$
\begin{array}{r}
x_{i}(k+1)=a_{i, 1} x_{1}(k)+\cdots+a_{i, n} x_{n}(k)+c_{i}, \\
\forall i=1,2, \ldots, n .
\end{array}
$$

Since system (17) is positive, we can obtain

$$
\begin{gathered}
a_{i, m} \geq 0, \quad \forall m=1,2, \ldots, n, \forall i=1,2, \ldots, n, \\
c_{i} \geq 0, \quad \forall i=1,2, \ldots, n .
\end{gathered}
$$

Hence, $\mathbf{A}_{p} \geq 0$ and $\mathbf{C}_{p} \geq 0, \forall p \in \mathscr{P}$.
Definition 3. System (1) with supply rate $\theta(k)=$ $\sum_{p=1}^{P}\left[\boldsymbol{\alpha}_{p} \mathbf{x}(k)+\beta_{p}\right]$, where $\boldsymbol{\alpha}_{p}$ are vectors with appropriate dimensions and $\beta_{p}$ are scalars, is said to be dissipative (strictly dissipative) if there exists a nonnegative function $S$ : $\mathbb{R}^{n} \rightarrow \mathbb{R}^{+}$with $S(0)=0$, called the storage function such that $\Delta S(k) \leq \theta(k)(\Delta S(k)<\theta(k))$.

Remark 4. In strictly dissipative case, $\Delta S(k)<\theta(k)$ can be expressed as $\Delta S(k) \leq-\rho S(k)+\theta(k)$, where $\rho>0$ provides a degree of freedom in choosing the quantity that we wish to dissipate. And if we enforce $\rho=0$, it becomes the dissipative case. Hence, in the rest of paper, we consider the general dissipative performance satisfying $\Delta S(k) \leq-\rho S(k)+\theta(k)$, where $\rho \geq 0$.

For switched positive system, the switched copositive function $S(k)=\mathbf{x}^{T}(k) \sum_{p=1}^{P} \eta_{p}(k) \mathbf{h}_{p}$, where $\mathbf{h}_{p} \geq \mathbf{0}, \forall p \in P$, can serve as a storage function. Then, the following theorem can be derived for the dissipativity of switched positive system (17) with respect to the particular supply rate $\theta(k)=$ $\sum_{p=1}^{P} \eta_{p}(k)\left[\boldsymbol{\alpha}_{p} \mathbf{x}(k)+\beta_{p}\right]$.

Theorem 5. Consider switched positive system (17), given a scalar $\rho \geq 0$. If there exists a set of vectors $\mathbf{h}_{p} \geq \mathbf{0}, \forall p \in P$, such that the following condition holds:

$$
\begin{gathered}
\mathbf{h}_{q}^{T} \mathbf{A}_{p}-(1-\rho) \mathbf{h}_{p}^{T}+\boldsymbol{\alpha}_{p} \preceq 0, \quad \forall(p, q) \in \mathscr{P} \times \mathscr{P}, \\
\mathbf{h}_{q}^{T} \mathbf{C}_{p}-\beta_{p} \leq 0, \quad \forall(p, q) \in \mathscr{P} \times \mathscr{P},
\end{gathered}
$$

then switched positive system (17) is dissipative with respect to a supply rate $\theta(k)=\sum_{p=1}^{P} \eta_{p}(k)\left[\boldsymbol{\alpha}_{p} \mathbf{x}(k)+\beta_{p}\right]$.

Proof. Given a set of vectors $\mathbf{h}_{p} \geq \mathbf{0}, \forall p \in \mathscr{P}$, the following switched copositive function is constructed:

$$
S(k)=\mathbf{x}^{T}(k) \sum_{p=1}^{P} \eta_{p}(k) \mathbf{h}_{p} .
$$

By the definition of dissipativity and supply rate $\theta(k)=$ $\sum_{p=1}^{P} \eta_{p}(k)\left[\boldsymbol{\alpha}_{p} \mathbf{x}(k)+\beta_{p}\right]$, we have to demonstrate the following inequality:

$$
\begin{aligned}
& \sum_{p=1}^{P}\left\{\eta_{p}(k+1) \mathbf{h}_{p}^{T} \mathbf{x}(k+1)-\eta_{p}(k)\right. \\
& \left.\quad \times\left[(1-\rho) \mathbf{h}_{p}^{T} \mathbf{x}(k)-\boldsymbol{\alpha}_{p} \mathbf{x}(k)+\beta_{p}\right]\right\} \leq 0,
\end{aligned}
$$

which ensures $\Delta S(k) \leq-\rho S(k)+\theta(k)$. As this has to be satisfied for arbitrary switching signals, it follows that (23) has to hold for any special configuration

$$
\begin{aligned}
\eta_{p}(k) & =1, & & \eta_{l \neq p}(k)=0, \\
\eta_{q}(k+1) & =1, & & \eta_{l \neq q}(k+1)=0,
\end{aligned}
$$

$\forall k \in \mathbb{N}$ and for all nonzero $\mathbf{x}(k) \in \mathbb{R}^{n}$. Hence (23) means

$$
\begin{array}{r}
\mathbf{h}_{q}^{T} \mathbf{x}(k+1)-(1-\rho) \mathbf{h}_{p}^{T} \mathbf{x}(k)+\boldsymbol{\alpha}_{p} \mathbf{x}(k)-\beta_{p} \leq 0, \\
\forall(p, q) \in \mathscr{P} \times \mathscr{P} .
\end{array}
$$


Using the system model as $\mathbf{x}(k+1)=\mathbf{A}_{p} \mathbf{x}(k)+\mathbf{C}_{p}$, one has

$$
\begin{array}{r}
{\left[\mathbf{h}_{q}^{T} \mathbf{A}_{p}-(1-\rho) \mathbf{h}_{p}^{T}+\boldsymbol{\alpha}_{p}\right] \mathbf{x}(k)+\mathbf{h}_{q}^{T} \mathbf{C}_{p}-\beta_{p} \leq 0,} \\
\forall(p, q) \in \mathscr{P} \times \mathscr{P} .
\end{array}
$$

From (21), it is easy to see that (26) is satisfied, which implies $\Delta S(k) \leq-\rho S(k)+\theta(k)$ and the dissipativity of system (17).

Theorem 5 provides us with a switched copositive function method in search for a set of vectors $\mathbf{h}_{p}, p \in \mathscr{P}$. If we particularly choose a common vector $\mathbf{h}=\mathbf{h}_{p}, \forall p \in \mathscr{P}$, the following corollary can be obtained.

Corollary 6. Consider switched positive system (17), given a scalar $\rho \geq 0$. If there exists a vector $\mathbf{h} \geq \mathbf{0}$ such that the following condition holds:

$$
\begin{gathered}
\mathbf{h}^{T} \mathbf{A}_{p}-(1-\rho) \mathbf{h}^{T}+\boldsymbol{\alpha}_{p} \preceq 0, \quad \forall p \in \mathscr{P}, \\
\mathbf{h}^{T} \mathbf{C}_{p}-\beta_{p} \leq 0, \quad \forall p \in \mathscr{P},
\end{gathered}
$$

then switched positive system (17) is dissipative with respect to supply rate $\theta(k)=\sum_{p=1}^{P} \eta_{p}(k)\left[\boldsymbol{\alpha}_{p} \mathbf{x}(k)+\beta_{p}\right]$.

Proof. Let $\mathbf{h}=\mathbf{h}_{p}, \forall p \in \mathscr{P}$ in Theorem 5; Corollary 6 can be proved.

Remark 7. It is obvious to see that conditions in Theorem 5 are less conservative than Corollary 6 due to the explicit fact that a common storage function yields more conservative results than multiple storage function. But, in order to meet the actual intersection system, a common storage function $S(k)=\mathbf{e}^{T} \mathbf{x}(k)$, where $\mathbf{e}^{T}=$ $\left[\begin{array}{llll}1 & 1 & 1 & 1\end{array}\right]$, is particularly chosen to describe the total lengths of the movement stored in the system; thus Corollary 6 will be used to solve the control problem for intersection system.

\section{Control Solution for \\ Two-Phase Intersection}

Now, based on the formulated control problem and analysis results in previous section, considering the two-phase intersection system, the feedback controller (8) has to be designed in which the state feedback gains $\mathbf{K}_{p}, p \in \mathscr{P}$, are the feedback gains needed to be determined. As what is discussed, to achieve a nonaccumulative closed loop with an available feedback controller, the design objective can be summarized as the following three points should be satisfied.

(1) Positivity. To ensure the availability of the feedback control in actual applications, we have that $\mathbf{x}(k) \geq 0$ holds for all $k \in$ $\mathbb{N}$ and any $\mathbf{x}(0) \geq 0$, that is, preserving the positivity of closed loop.

(2) Dissipativity. Given the storage function $S(k)=\mathbf{e}^{T} \mathbf{x}(k)$, where $\mathbf{e}^{T}=\left[\begin{array}{llll}1 & 1 & 1 & 1\end{array}\right]$, the closed loop should be dissipative with respect to supply rate $\theta(k)=\sum_{p=1}^{2} \eta_{p}(k)\left[\boldsymbol{\alpha}_{p} \mathbf{x}(k)+\beta_{p}\right]$, where $\boldsymbol{\alpha}_{p}$ and $\beta_{p}$ are defined in (15), which makes the intersection system nonaccumulative.

(3) Control Constraint. The constraint on the green time has to be satisfied to avoid unacceptable stop time for drivers in other approaches. By $0 \leq g_{p}(k) \leq g_{p \text {, max }}$ and $g_{p}(k)=$ $u_{p}(k)=\mathbf{K}_{p} \mathbf{x}(k)$, the following constraint must be satisfied $0 \leq \mathbf{K}_{p} \mathbf{x}(k) \leq g_{p \text {, max }}$.

Considering the closed loop system (9), the first point concerned with positivity can be solved through Lemma 2 for the switched positive system; that is, $\mathbf{A}_{p} \geq 0, \forall p \in \mathscr{P}$, and $\mathbf{C}_{p} \succeq 0, \forall p \in \mathscr{P}$, should be satisfied. Obviously $\mathbf{C}_{p} \geq 0$, $\forall p \in P$, is automatically satisfied for two-phase intersection system since $s_{p}>0, \forall p \in \mathscr{P}$ and $L>0$. Moreover, $\mathbf{A}_{p} \geq 0$ can be rewritten as

$$
\mathbf{I}+\mathbf{B}_{p} \mathbf{K}_{p} \geq 0, \quad \forall p \in \mathscr{P},
$$

to preserve positivity of feedback closed loop system.

Then, the second point on dissipativity can be solved by Corollary 6 where the common vector is chosen as $\mathrm{e}^{T}=$ $\left[\begin{array}{llll}1 & 1 & 1 & 1\end{array}\right]$. Firstly, we have

$$
\begin{aligned}
& \mathbf{e}^{T} \mathbf{C}_{1}-\beta_{1}=\left[\begin{array}{llll}
1 & 1 & 1 & 1
\end{array}\right]\left[\begin{array}{llll}
s_{1} L & s_{2} L & 0 & 0
\end{array}\right]^{T}-\left(s_{1}+s_{2}\right) L=0, \\
& \mathbf{e}^{T} \mathbf{C}_{2}-\beta_{2}=\left[\begin{array}{llll}
1 & 1 & 1 & 1
\end{array}\right]\left[\begin{array}{lllll}
0 & 0 & s_{3} L & s_{4} L
\end{array}\right]^{T}-\left(s_{3}+s_{4}\right) L=0 .
\end{aligned}
$$

Thus, it yields that $\mathbf{e}^{T} \mathbf{C}_{p}-\beta_{p} \leq 0, \forall p \in \mathscr{P}$, is satisfied for intersection system.

Moreover, to ensure dissipativity, the following condition has to be satisfied:

$$
\mathbf{e}^{T}\left(\mathbf{I}+\mathbf{B}_{p} \mathbf{K}_{p}\right)-(1-\rho) \mathbf{e}^{T}+\boldsymbol{\alpha}_{p} \preceq 0, \quad \forall p \in \mathscr{P},
$$

where $\boldsymbol{\alpha}_{p}=\mathbf{K}_{p}\left(\sum_{i=1}^{4} q_{i}-d_{p}\right), d_{1}=s_{1}+s_{2}$, and $d_{2}=s_{3}+s_{4}$.

At last, the constraint on the feedback is considered. Note that queue length $x_{i}$ belongs to the region of admissible states as $\mathscr{X}_{i}=\left\{x \mid 0 \leq x_{i} \leq x_{i, \max }\right\}$; we denote set $\mathscr{X}=\{\mathbf{x} \mid 0 \preceq \mathbf{x} \preceq$ $\left.\mathbf{x}_{\max }\right\}$, where $\mathbf{x}_{\max }=\left[\begin{array}{llll}x_{1, \max } & x_{2, \max } & x_{3, \max } & x_{4, \max }\end{array}\right]^{T}$. Given any $\mathbf{x}_{1}, \mathbf{x}_{2} \in \mathscr{X}$ and $0 \leq \alpha \leq 1$, we have $0 \preceq \alpha \mathbf{x}_{1}+(1-\alpha) \mathbf{x}_{2} \preceq$ $\mathbf{x}_{\max }$, so $\mathscr{X}$ is a convex set with 16 vertices which are denoted as $\mathbf{v}_{1}, \mathbf{v}_{2}, \ldots, \mathbf{v}_{16}$.

Furthermore, the constraint set on the green time can be expressed by set $\mathscr{U}_{p}=\left\{\mathbf{x} \mid 0 \leq \mathbf{K}_{p} \mathbf{x}(k) \leq g_{p \text {, max }}, \mathbf{x} \in \mathscr{X}\right\}$. Since $\mathscr{X}$ is convex, we only need to check that the vertices $v_{1}, v_{2}, \ldots, v_{16}$ are included in control constraint $U_{p}$; that is,

$$
0 \leq \mathbf{K}_{p} \mathbf{v}_{i} \leq g_{p, \text { max }}, \quad \forall p \in \mathscr{P}, \forall i=1,2, \ldots, 16 .
$$

Summarizing above discussion, a solution for nonaccumulative feedback control is presented as follows.

Proposition 8. Consider the two-phase intersection system (5), given a scalar $\rho \geq 0$. If there exist two feedback gains $\mathbf{K}_{1}$ and $\mathbf{K}_{2}$ such that

$$
\begin{gathered}
\mathbf{I}+\mathbf{B}_{p} \mathbf{K}_{p} \geq 0, \quad \forall p \in \mathscr{P}, \\
\mathbf{e}^{T}\left(\mathbf{I}+\mathbf{B}_{p} \mathbf{K}_{p}\right)-(1-\rho) \mathbf{e}^{T}+\boldsymbol{\alpha}_{p} \preceq 0, \quad \forall p \in \mathscr{P}, \\
0 \leq \mathbf{K}_{p} \mathbf{v}_{i} \leq g_{p, \text { max }}, \quad \forall p \in \mathscr{P}, \forall i=1,2, \ldots, 16,
\end{gathered}
$$




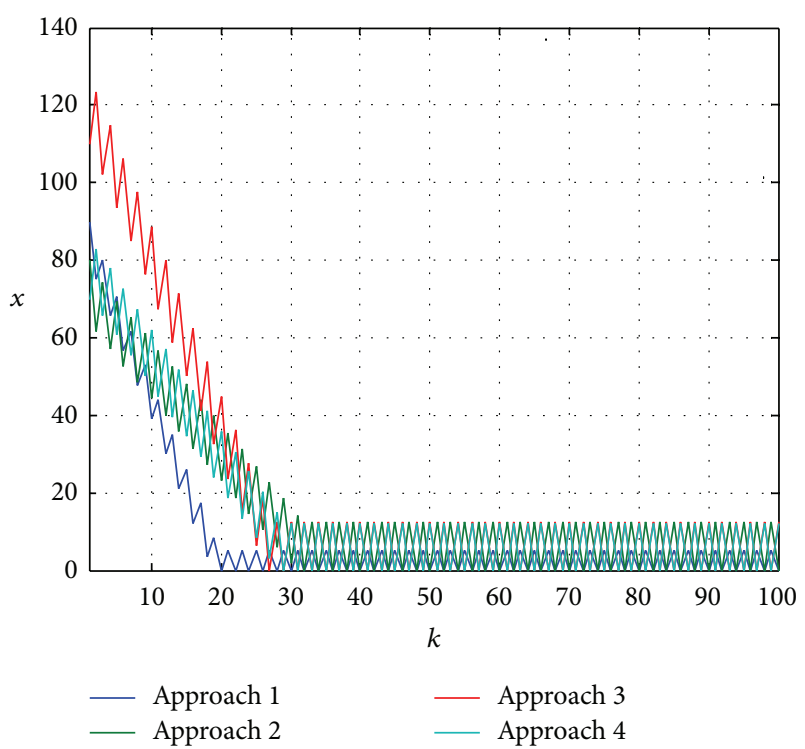

FIGURE 2: Evolution of queues within dissipative closed loop system.

where vectors $\boldsymbol{\alpha}_{p}$ and $\mathbf{v}_{i}$ are defined in (15) and (31), respectively, the green times chosen as $g_{p}(k)=\mathbf{K}_{p} \mathbf{x}(k), p \in \mathscr{P}$, can guarantee the nonaccumulative performance of the closed loop system (9).

Remark 9. In actual applications, a lower boundary for the effective green time has to be selected as $g_{p \text {, min }}$, thus the operation of the controller for each $k$ is considered in the following form:

$$
g_{p}(k)= \begin{cases}\mathbf{K}_{p} \mathbf{x}(k) & \text { if } g_{p}(k)>g_{p, \min } \\ g_{p, \text { min }} & \text { if } g_{p}(k) \leq g_{p, \min }\end{cases}
$$

Here, it should be noted that since $\mathbf{x}(k)$ decrease along with the time to relieve the oversaturated queue, $g_{p}(k)$ will finally converge to $g_{p, \min }$. Thus, $g_{p, \min }$ can be often chosen as an appropriate time for undersaturated situations.

Remark 10. Proposition 8 is an LP problem, which can be numerically solved by the LP optimal toolbox.

Example 11. Consider a two-phase intersection as $s_{1}=$ $0.4 \mathrm{veh} / \mathrm{s}, s_{2}=0.6 \mathrm{veh} / \mathrm{s}, s_{3}=0.7 \mathrm{veh} / \mathrm{s}$, and $s_{4}=0.6 \mathrm{veh} / \mathrm{s}$, $q_{1}=0.2 \mathrm{veh} / \mathrm{s}, q_{2}=0.1 \mathrm{veh} / \mathrm{s}, q_{3}=0.15 \mathrm{veh} / \mathrm{s}$, and $q_{4}=$ $0.25 \mathrm{veh} / \mathrm{s}$, and $g_{p, \max }=100 \mathrm{~s}, g_{p, \text { min }}=50 \mathrm{~s}$. Given an initial oversaturated queue $\mathbf{x}^{T}(0)=\left[\begin{array}{llll}90 & 80 & 110 & 70\end{array}\right]$, the simulation results are shown in Figure 2.

From the simulation results in Figure 2, we see that the oversaturated situation can be relieved by state feedback control, and we see that, after $k=30$, the intersection runs in an undersaturated situation.

\section{Extension to Multiphase Intersection}

In this section, the results in previous section based on dissipativity and positivity of positive switched system will be generalized to multiphase intersection. At first, the system model for multiphase intersection will be presented as follows.

It is assumed that there exist $P$ phases for the intersection with $n$ movements. Hereby, the following sets are defined. For each phase $p \in \mathscr{P}$, concerned with movements, we define $\mathscr{I}=\{1,2, \ldots, M\}$, the activated movement set $\mathscr{I}_{a}$, and unactivated movement set $\overline{\mathscr{F}}_{a}$; similarly we have $\mathscr{I}_{a} \cap \overline{\mathscr{F}}_{a}=$ $\varnothing$ and $\mathscr{I}_{a} \cup \overline{\mathscr{I}}_{a}=\mathscr{I}$.

In accordance with the modeling procedure for twophase intersection, in each phase $p \in \mathscr{P}$, the queue length of movements $x_{i}(k), i \in \mathscr{I}$, evolves according to

$$
x_{i}(k+1)=x_{i}(k)+A_{i, g_{p}}-D_{i, g_{p}} .
$$

Similar to the derivation of two-phase intersection model, we have

$$
\begin{gathered}
A_{i, g_{p}}=q_{i} g_{p}, \quad i \in \mathscr{I}_{a} \cup \overline{\mathscr{I}}_{a}=\mathscr{I} \\
D_{i, g_{p}}= \begin{cases}s_{i}\left(g_{p}-L\right) & i \in I_{a} \\
0 & i \in \bar{I}_{a} .\end{cases}
\end{gathered}
$$

Thus, the evolution of movement $i \in \mathscr{I}$ in phase $p \in \mathscr{P}$ is described as follows:

$$
x_{i}(k+1)= \begin{cases}x_{i}(k)+\left(q_{i}-s_{i}\right) g_{p}+s_{i} L & i \in \mathscr{I}_{a} \\ x_{i}(k)+q_{i} g_{p} & i \in \overline{\mathscr{F}}_{a} .\end{cases}
$$

With a switching signal $\sigma(k)$ among subsystems $p \in \mathscr{P}$, define $\eta_{p}(k)$ same as in (4). The above equations can be restated in state space form

$$
\begin{array}{r}
\mathbf{x}(k+1)=\sum_{p=1}^{P} \eta_{p}(k)\left[\mathbf{x}(k)+\mathbf{B}_{p} u_{p}(k)+\mathbf{C}_{p}\right], \\
0 \leq u_{p}(k) \leq g_{p, \max },
\end{array}
$$

where

$$
\begin{gathered}
\mathbf{x}(k)=\left[\begin{array}{c}
x_{1}(k) \\
x_{2}(k) \\
\vdots \\
x_{n}(k)
\end{array}\right], \quad \mathbf{B}_{p}=\left[\begin{array}{c}
b_{1} \\
b_{2} \\
\vdots \\
b_{n}
\end{array}\right], \\
b_{i}=\left\{\begin{array}{ll}
q_{i}-s_{i} & i \in \mathscr{I}_{a} \\
q_{i} & i \in \mathscr{\mathscr { I }}_{a},
\end{array} \quad \mathbf{C}_{p}=\left[\begin{array}{c}
c_{1} \\
c_{2} \\
\vdots \\
c_{n}
\end{array}\right],\right. \\
c_{i}=\left\{\begin{array}{ll}
s_{i} L & i \in \mathscr{I}_{a} \\
0 & i \in \mathscr{I}_{a},
\end{array} \quad u_{p}(k)=g_{p}(k) .\right.
\end{gathered}
$$

The phases are working in turns in the intersection system model; the switching signal $\sigma(k)$ is defined as

$$
\sigma(k)=p \quad \text { if } \sigma(k-1)=q, \quad \text { where } p \neq q, p, q \in \mathscr{P},
$$

which implies that the switching occurs at each instant $k$. 
With the aid of state feedback control as

$$
u_{p}(k)=\sum_{p=1}^{P} \eta_{p}(k) \mathbf{K}_{p} \mathbf{x}(k), \quad 0 \leq u_{p}(k) \leq g_{p, \max }
$$

the closed loop of intersection system is expressed as follows:

$$
\begin{array}{r}
\mathbf{x}(k+1)=\sum_{p=1}^{P} \eta_{p}(k)\left[\mathbf{A}_{p} \mathbf{x}(k)+\mathbf{C}_{p}\right], \\
0 \leq \mathbf{K}_{p} \mathbf{x}(k) \leq g_{p \text {, max }},
\end{array}
$$

where $\mathbf{A}_{p}=\mathbf{I}+\mathbf{B}_{p} \mathbf{K}_{p}$.

Furthermore, following philosophy of positive and dissipative control for intersection in previous section, the positivity, dissipativity, and control constraint are guaranteed by the following steps.

Positivity. Based on Lemma 2, it is easy to see that the following condition is required to ensure the positivity:

$$
\mathbf{I}+\mathbf{B}_{p} \mathbf{K}_{p} \geq 0, \quad \forall p \in \mathscr{P} .
$$

Dissipativity. Since the storage function is $S(k)=\mathbf{e}^{T} \mathbf{x}(k)$, where $\mathbf{e}^{T}=\left[\begin{array}{llll}1 & 1 & \cdots & 1\end{array}\right]$, and supply rate is

$$
\theta(k)=\sum_{p=1}^{P} \eta_{p}(k) \theta_{p}(k)=\sum_{p=1}^{P} \eta_{p}(k)\left[A_{p}(k)-D_{p}(k)\right],
$$

where $A_{p}(k)=g_{p}(k) \sum_{i=1}^{P} q_{i}=\mathbf{K}_{p} \mathbf{x}(k) \sum_{i=1}^{P} q_{i}$ and $D_{p}(k)=$ $\sum_{i \in \mathscr{I}_{a}} s_{i}\left(g_{p}-L\right)=\sum_{i \in \mathscr{I}_{a}} s_{i}\left[\mathbf{K}_{p} \mathbf{x}(k)-L\right]$. Furthermore, by letting $\boldsymbol{\alpha}_{p}=\mathbf{K}_{p}\left(\sum_{i=1}^{P} q_{i}-d_{p}\right), \beta_{p}=d_{p} L$, where $d_{p}=\sum_{i \in \mathscr{I}_{a}} s_{i}$, we get $\theta(k)=\sum_{p=1}^{P} \eta_{p}(k)\left[\boldsymbol{\alpha}_{p} \mathbf{x}(k)+\beta_{p}\right]$.

By $\mathbf{e}^{T} \mathbf{C}_{p}-\beta_{1}=\left[\begin{array}{llll}1 & 1 & \cdots & 1\end{array}\right]\left[\begin{array}{llll}c_{1} & c_{2} & \cdots & c_{n}\end{array}\right]^{T}-$ $L \sum_{i \in \mathcal{F}_{a}} s_{i}=0$, we can obtain $\mathbf{e}^{T} \mathbf{C}_{p}-\beta_{p} \leq 0, \forall p \in \mathscr{P}$, for multiphase intersection. Then, on the basis of Corollary 6 , given a scalar $\rho \geq 0$, the following condition should be satisfied to ensure the dissipativity:

$$
\mathbf{e}^{T}\left(\mathbf{I}+\mathbf{B}_{p} \mathbf{K}_{p}\right)-(1-\rho) \mathbf{e}^{T}+\boldsymbol{\alpha}_{p} \preceq 0, \quad \forall p \in \mathscr{P} .
$$

Control Constraint. Each movement $x_{i}$ belongs to $\mathscr{X}_{i}=\{x \mid$ $\left.0 \leq x_{i} \leq x_{i, \max }\right\}$, and define convex set as $\mathscr{X}=\{\mathbf{x} \mid 0 \preceq \mathbf{x} \preceq$ $\left.\mathbf{x}_{\max }\right\}$, where $\mathbf{x}_{\text {max }}=\left[\begin{array}{llll}x_{1, \max } & x_{2, \max } & \cdots & x_{n, \max }\end{array}\right]^{T}$; it is easy to see that there are $N=n^{2}$ vertices of $\mathscr{X}$ which are denoted as $\mathbf{v}_{1}, \mathbf{v}_{2}, \ldots, \mathbf{v}_{N}$. From the constraint set on the green time by set $\mathscr{U}_{p}=\left\{\mathbf{x} \mid 0 \leq \mathbf{K}_{p} \mathbf{x}(k) \leq g_{p \text {, max }}, \mathbf{x} \in \mathscr{X}\right\}$, the following condition has to be satisfied:

$$
0 \leq \mathbf{K}_{p} \mathbf{v}_{i} \leq g_{p, \max }, \quad \forall p \in \mathscr{P}, \forall i=1,2, \ldots, N
$$

Summarizing the above steps, the nonaccumulative feedback control solution multiphase intersection is presented as follows.
Proposition 12. Consider the P-phase intersection system (37), given a scalar $\rho \geq 0$. If there exist $p$ feedback gains $\mathbf{K}_{p}$, $p \in \mathscr{P}$, such that

$$
\begin{gathered}
\mathbf{I}+\mathbf{B}_{p} \mathbf{K}_{p} \geq 0, \quad \forall p \in \mathscr{P}, \\
\mathbf{e}^{T}\left(\mathbf{I}+\mathbf{B}_{p} \mathbf{K}_{p}\right)-(1-\rho) \mathbf{e}^{T}+\boldsymbol{\alpha}_{p} \preceq 0, \quad \forall p \in \mathscr{P}, \\
0 \leq \mathbf{K}_{p} \mathbf{v}_{i} \leq g_{p, \text { max }}, \quad \forall p \in \mathscr{P}, \quad \forall i=1,2, \ldots, N,
\end{gathered}
$$

where vectors $\boldsymbol{\alpha}_{p}$ and $\mathbf{v}_{i}$ are defined in (44) and (45), respectively, the green times chosen as $g_{p}(k)=\mathbf{K}_{p} \mathbf{x}(k), p \in \mathscr{P}$, can guarantee the nonaccumulative performance of the closed loop system (41).

\section{Conclusions}

By modeling the intersection into positive switched system, a dissipativity-based control strategy is proposed for online traffic signalization in this paper. Through fulfilling the positivity, dissipativity, and control constraint, an LP problem based design method is presented. A numerical example is provided to illustrate our results, and, furthermore, the twophase intersection results are extended to multiphase intersection. The positive switched system approach provides us with a new insight on modeling intersection; introducing other advanced control schemes from positive switched system to intersection system is our future work.

\section{Conflict of Interests}

The authors declare that there is no conflict of interests regarding the publication of this paper.

\section{References}

[1] M. Kaltenbach and H. Koivo, "Modelling and control of urban traffic flow," in Proceedings of the Joint Automatic Control Conference, pp. 147-154, University of Texas, Houston, Tex, USA, 1974.

[2] Y. Tangsheng, On line traffic signalization using robust feedback control [Ph.D. thesis], Faculty of the Virginia Polytechnic Institute and State University, Virginia, Va, USA, 1997.

[3] C. Diakaki, M. Papageorgiou, and K. Aboudolas, "A multivariable regulator approach to traffic-responsive network- Wide signal control," Control Engineering Practice, vol. 10, no. 2, pp. 183-195, 2002.

[4] D. Gazis, "Optimal control of a system of oversaturated intersections," Operations Research, vol. 12, pp. 815-831, 1964.

[5] D. Gazis and R. Potts, "The oversaturated intersection," in Proceedings of the 2nd International Symposium on the Theory of Road Traffic Flow Organization for Economic Cooperation and Development, pp. 221-237, Paris, France, 1965.

[6] D. Green, "Control of oversaturated intersections," Operational Research Quarterly, vol. 18, no. 2, pp. 161-173, 1967.

[7] G. C. D’Ans and D. C. Gazis, "Optimal control of oversaturated store-and-forward transportation networks," Transportation Science, vol. 10, no. 1, pp. 1-19, 1976.

[8] T. Chang and J. Lin, "Optimal signal timing for an oversaturated intersection," Transportation Research Part B, vol. 34, no. 6, pp. 471-491, 2000. 
[9] B. de Schutter and B. de Moor, "Optimal traffic light control for a single intersection," European Journal of Control, vol. 4, no. 3, pp. 260-276, 1998.

[10] F. Motawej, R. Bouyekhf, and A. El Moudni, "A dissipativitybased approach to traffic signal control for an over-saturated intersection," Journal of the Franklin Institute, vol. 348, no. 4, pp. 703-717, 2011.

[11] F. Boillot, J. M. Blosseville, J. B. Lesort, V. Motyka, M. Papageorgiou, and S. Sellam, "Optimal signal control of urban traffic networks," in Proceedings of the 6th IEE International conference on Road Traffic Monitoring and Control, pp. 75-79, London, UK, April 1992.

[12] F. Dion and S. Yagar, "Real-time control of signalized networks different approaches for different needs," in Proceedings of the 8th IEE International Conference on Road Traffic Monitoring and Control, pp. 56-60, London, UK, 1996.

[13] P. B. Hunt, D. I. Robertson, and R. D. Bretherton, "The SCOOT on-line traffic signal optimisation technique," Traffic Engineering \& Control, vol. 23, no. 4, pp. 190-192, 1982.

[14] R. A. Decarlo, M. S. Branicky, S. Pettersson, and B. Lennartson, "Perspectives and results on the stability and stabilizability of hybrid systems," Proceedings of the IEEE, vol. 88, no. 7, pp. 10691082, 2000.

[15] H. Lin and P. J. Antsaklis, "Stability and stabilizability of switched linear systems: a survey of recent results," IEEE Transactions on Automatic Control, vol. 54, no. 2, pp. 308-322, 2009.

[16] W. Xiang and J. Xiao, "Stabilization of switched continuoustime systems with all modes unstable via dwell time switching," Automatica, vol. 50, no. 3, pp. 940-945, 2014.

[17] W. Xiang and J. Xiao, "Convex sufficient conditions on asymptotic stability and $\ell_{2}$ gain performance for uncertain discretetime switched linear systems," IET Control Theory \& Applications, vol. 8, no. 3, pp. 211-218, 2014.

[18] W. Xiang and J. Xiao, " $H_{\infty}$ finite-time control for switched nonlinear discrete-time systems with norm-bounded disturbance," Journal of the Franklin Institute, vol. 348, no. 2, pp. 331-352, 2011.

[19] W. Xiang and J. Xiao, "Stability analysis and control synthesis of switched impulsive systems," International Journal of Robust and Nonlinear Control, vol. 22, no. 13, pp. 1440-1459, 2012.

[20] W. Xiang, J. Xiao, and M. N. Iqbal, "Asymptotic stability, $\ell_{2}$ gain, boundness analysis, and control synthesis for switched systems: a switching frequency approach," International Journal of Adaptive Control and Signal Processing, vol. 26, no. 4, pp. 350373, 2012.

[21] W. Xiang, J. Xiao, and M. N. Iqbal, "Robust observer design for nonlinear uncertain switched systems under asynchronous switching," Nonlinear Analysis: Hybrid Systems, vol. 6, no. 1, pp. 754-773, 2012.

[22] X. Zhao, L. Zhang, P. Shi, and M. Liu, "Stability of switched positive linear systems with average dwell time switching," Automatica, vol. 48, no. 6, pp. 1132-1137, 2012.

[23] X. Zhao, X. Liu, S. Yin, and H. Li, "Improved results on stability of continuous-time switched positive linear systems," Automatica, vol. 50, no. 2, pp. 614-621, 2014.

[24] X. Zhao, S. Yin, H. Li, and B. Niu, "Switching stabilization for a class of slowly switched systems," IEEE Transactions on Automatic Control, no. 99, 1 page, 2014. 


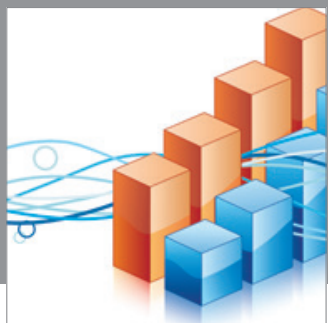

Advances in

Operations Research

mansans

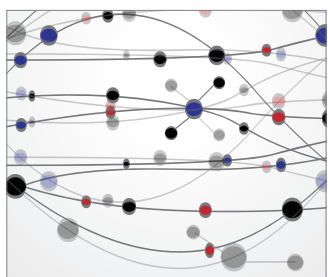

The Scientific World Journal
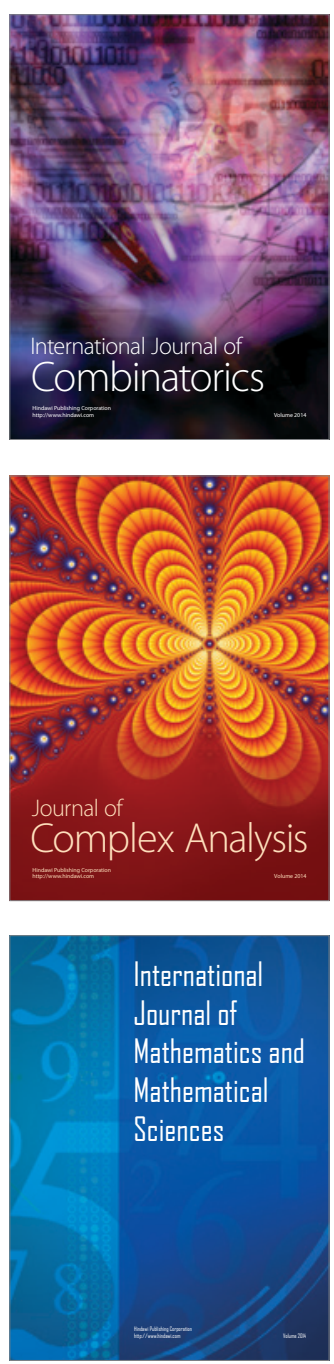
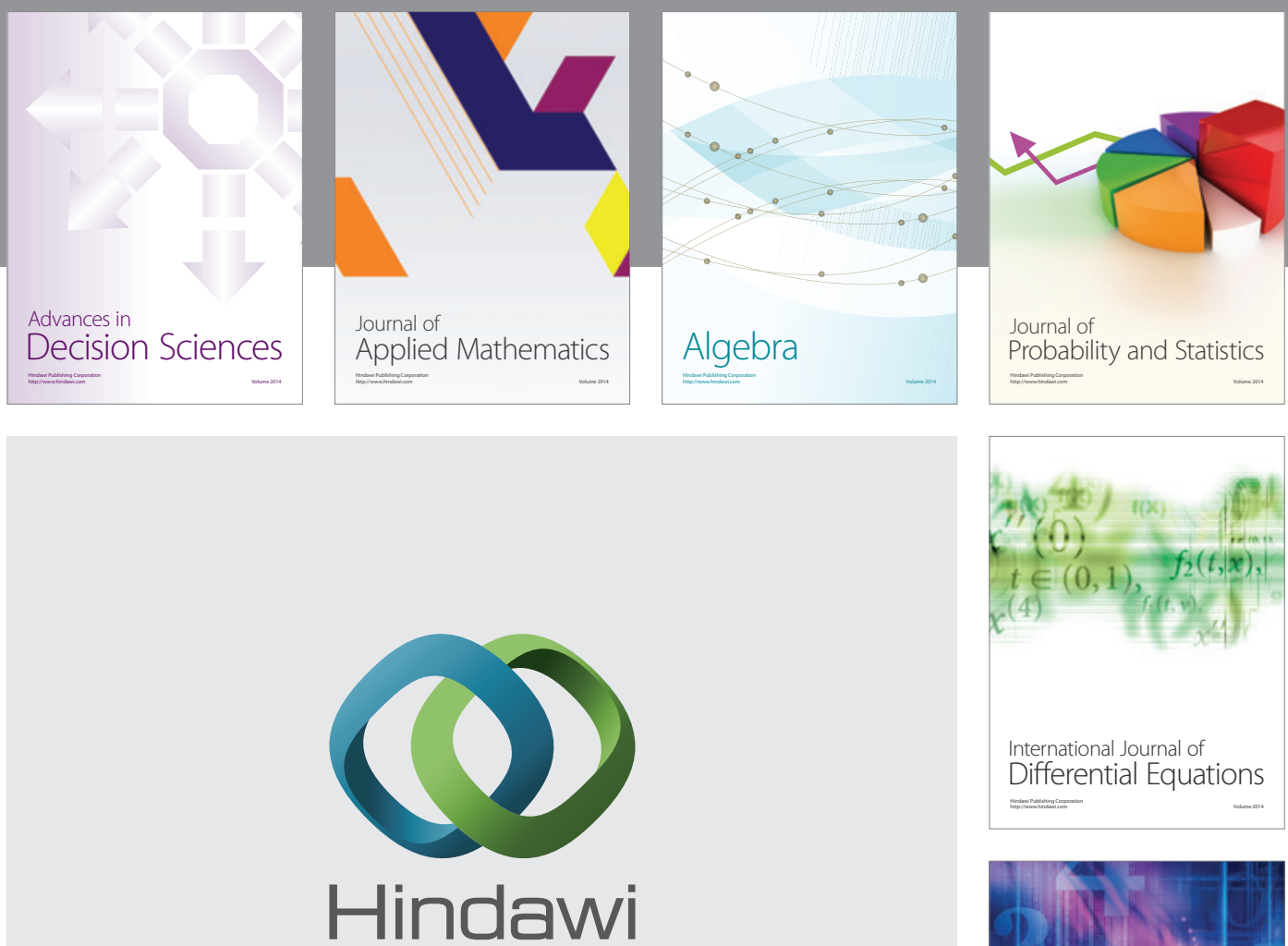

Submit your manuscripts at http://www.hindawi.com
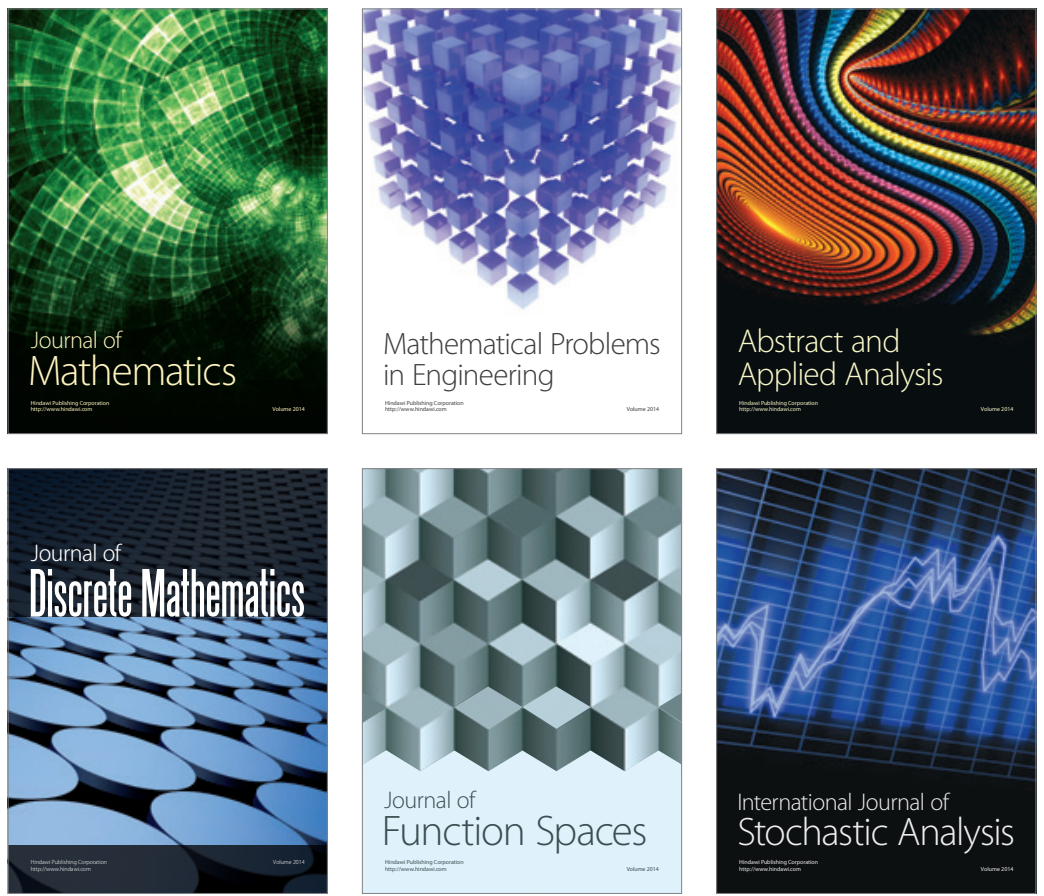

Journal of

Function Spaces

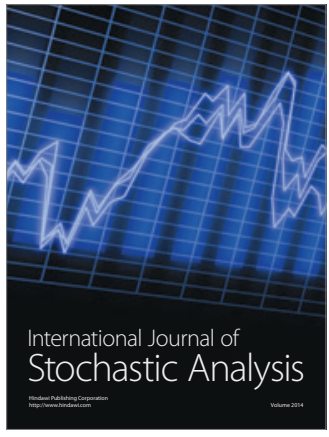

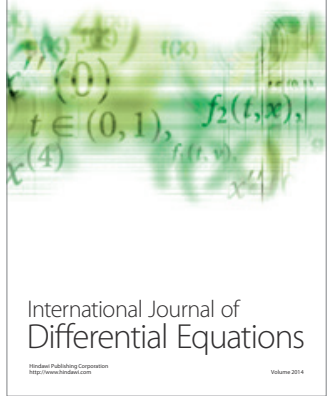
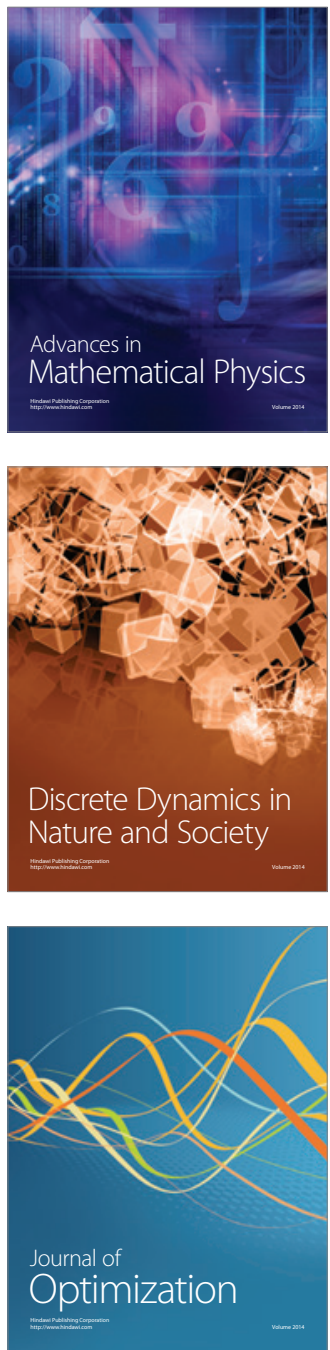